\title{
Aerodynamic Characteristics of Helicopter with Ducted Fan Tail Rotor in Hover under Low-Speed Crosswind
}

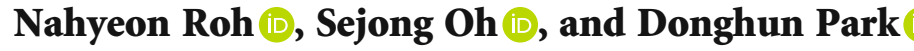 \\ Aerospace Engineering, Pusan National University, 46241, Republic of Korea \\ Correspondence should be addressed to Donghun Park; parkdh@pusan.ac.kr
}

Received 21 February 2020; Revised 2 September 2020; Accepted 9 October 2020; Published 27 October 2020

Academic Editor: Hao J. Xia

Copyright (C) 2020 Nahyeon Roh et al. This is an open access article distributed under the Creative Commons Attribution License, which permits unrestricted use, distribution, and reproduction in any medium, provided the original work is properly cited.

\begin{abstract}
The tail rotor of a helicopter operating under low-speed crosswind undergoes highly complex flow due to the interaction between the main rotor, fuselage, and tail rotor system. In this study, numerical simulations have been conducted on the complete configuration of a helicopter with a ducted fan tail rotor system (comprising a main rotor, ducted fan tail rotor, fuselage, and empennage) to analyze the wake interaction in hovering flight under various crosswind directions. The flow characteristics around the tail rotor, the tail rotor thrust, and the yawing moment of the helicopter are investigated and evaluated. The aerodynamic forces are compared with those of a helicopter with an open-type tail rotor. The results indicate that the aerodynamic performance of the ducted fan tail rotor is highly affected by the wakes of both the main rotor and port wing. Nevertheless, the helicopter with a ducted fan tail rotor is observed to be much more directionally stable under various crosswind directions, than that with an open-type tail rotor. This is because the rotor is protected by the fixed part of the tail rotor system in the former case.
\end{abstract}

\section{Introduction}

A helicopter operates in a highly complex and unsteady flowfield due to the substantial interference among its components. The complex flow can significantly influence performance, stability, and handling quality. Notably, the performance of the tail rotor, which governs directional stability, is influenced considerably by the wake and vortex generated by the main rotor, fuselage, and incoming flow. In particular, several helicopters that comprise a main rotor and open-type tail rotor exhibit limitations in direction control at low-speeds [1]. In this condition, the tail rotor experiences rapid variation in yaw control effectiveness for marginal variations in the crosswind direction. The Federal Aviation Administration (FAA) Advisory Circular states that loss of tail rotor effectiveness (LTE) is a critical aerodynamic characteristic, which can result in the loss of aircraft control [2]. In this regard, LTE is one of the prominent causes of helicopter accidents. A total of 82 out of 547 accidents between 1993 and 2004 involved LTE during hovering and lowspeed flights [3].
Several studies have been conducted to investigate the aerodynamic characteristics of a complete helicopter configuration in hovering flight under crosswind. Amer and Gessow [4] indicated a region of difficulty of tail-rotor control, based on theoretically derived charts, which was then verified through flight tests. The charts indicated that directional control could be challenging under a low-speed crosswind. The U.S. Army conducted an experimental study to evaluate the LTE for OH-58 at speeds ranging from 20 to 45 knots under crosswind from various directions [5]. Through flight tests at 30 knots, Ellin $[6,7]$ identified and divided the flight envelope of the Lynx helicopter into six regions with different mechanisms of main/tail rotor interaction. Recently, Thiemeier et al. [8] conducted a numerical study of a RACER (Airbus Helicopters) in hovering condition, under the influence of low-speed crosswinds from eight directions. They verified that wake-wake and wake-airframe interactions substantially impact the efficiency and controllability of a helicopter. The aforementioned studies verified that a crosswind at a low speed rather than a high speed critically impacts the performance of an open-type tail rotor and 
causes directional stability problems in the hovering condition.

Apart from the conventional open-type tail rotor, a ducted fan-type tail rotor (first proposed by Aerospatiale [9, 10]) has been developed and increasingly applied to various helicopters. The ducted fan tail rotor comprises fixed parts (shroud, outer shroud, hub, and vertical fin) that are exposed to the external flow and a rotating part (rotor) that is protected by the fixed parts. It has been established that, in general, a ducted fan tail rotor provides advantages in terms of safety by preventing tail rotor strike events. Moreover, the suppression of the tip vortex, alleviation of the compressibility effect, and noise reduction are known additional benefits $[9,10]$. Additionally, the ducted fan tail rotor can exhibit characteristics different from those of an open-type tail rotor (because of its geometric features) as follows. The interaction with the main rotor and fuselage can result in different influences on the tail rotor performance. Furthermore, the fixed parts which are completely separate from the tail rotor can produce significant force in comparison with the vertical fin of conventional helicopters. Therefore, it is essential to consider the complete configuration for analyzing the aerodynamic characteristics of helicopters with a ducted fan tail rotor. However, only a few studies have been conducted on the complete configuration of a helicopter with a ducted fan tail rotor. Most of the previous studies have focused only on isolated ducted fan tail rotors [11-15]. In particular, Alpman et al. $[16,17]$ conducted numerical studies on the fuselage of Comanche with a ducted fan tail rotor by simulating the tail rotor as a uniform actuator disk. Nevertheless, the main rotor and its influence were not considered in their study.

Several challenging problems are encountered in the numerical simulation of the complete configuration with a ducted fan tail rotor under various flight conditions. First, the generation of the wall-adapted mesh is a timeconsuming task. In particular, mesh generation for the ducted fan tail rotor, which has many blades and a gap between the blade tip and shroud, is highly complex. Second, there is a time-scale issue in the performance of the transient simulation for both the main rotor and tail rotor simultaneously. This is because the tail rotor rotates approximately ten times faster than the main rotor. If the time-step size is selected to correspond to a $1^{\circ}$ rotation of the tail rotor, the main rotor rotates by approximately $0.1^{\circ}$ for each time-step. Therefore, substantial computational time and cost are required to obtain adequate revolutions of the main rotor for analyzing the wake interference between the two rotors.

In this study, numerical analysis has been conducted for the complete configuration of a helicopter, including the main rotor, ducted fan tail rotor, and fuselage. For improving numerical efficiency and alleviating the time-scale issue, actuator surface and disk methods are employed to simulate the effect of the main rotor and tail rotor, respectively. The hovering condition with various crosswind directions is considered to investigate the flow characteristics at low-speed crosswind. The objectives of the present study are as follows: (1) identification of the flow-field characteristics around a ducted fan tail rotor under the interactions among the main rotor, tail rotor, and body, (2) evaluation of the average/fluctuation of forces and yawing moment with respect to the crosswind direction, and (3) comparison of the aerodynamic performance characteristics with those of the open-type tail rotor.

The rest of this paper is organized as follows: approaches for performing numerical analysis are discussed in Section 2, including details of the geometry, analysis condition, grid generation, numerical methods, and their validations. Comparisons and examination of the numerical results are presented in Section 3. The concluding remarks are presented in Section 4.

\section{Analysis Method}

2.1. Geometry and Analysis Conditions. In this study, the configuration of H155 [18] of Airbus Helicopters is considered for the numerical analysis (see Figure 1). The surface geometry of the airframe is cleaned up for convenient grid generation. The engine intake and exhaust are omitted for simplicity. Figure 2 depicts the resulting surface geometry and the designated name of each part. The rear section is defined as the tail rotor system, which is divided into a fixed part and rotating part. Hereafter, the rotating part (tail rotor disk) is denoted as the tail rotor. Table 1 presents the parameters and specifications of the main and tail rotors. The main rotor rotates in a clockwise direction when viewed from the top. Hence, the tail rotor produces thrust in the direction toward the port side. The tail rotor rotates in the top-aft direction (see Figure 3), indicating that the blades at the top move toward the rearward direction. All the blades are assumed to be rigid. The collective pitch angles of the main and tail rotors are set to the hovering flight condition.

To investigate the variations in aerodynamic performance with respect to crosswinds, crosswinds from 12 directions are considered. The directions of crosswind are selected from $0^{\circ}$ to $360^{\circ}$ at intervals of $30^{\circ}$ (see Figure 4). The crosswind speed is fixed at 20 knots, which was established as a critical speed in terms of the sensitivity of the tail rotor's aerodynamic performance [1].

2.2. Numerical Methods. The actuator methods enable timeand cost-efficient analysis for flow problems, including those involving rotating blades. The methods replace the effect of rotor blades with equivalent momentum source terms that are imposed on the cells corresponding to the rotor blade/disk. In the actuator disk method (ADM), the momentum source is inserted into the fixed cells corresponding to the rotor disk. In the actuator surface method (ASM), the momentum source is added only to the cells corresponding to the blades whose location varies with time according to the rotation. The use of the actuator methods can considerably reduce the total number of cells required because these methods eliminate the need for generating body-fitted grids for blades, thereby reducing the time required for the preprocessing. Furthermore, this may lead to an additional reduction in the computational cost because these methods do not require computationally expensive sliding or an overset mesh technique to simulate rotating blades. 


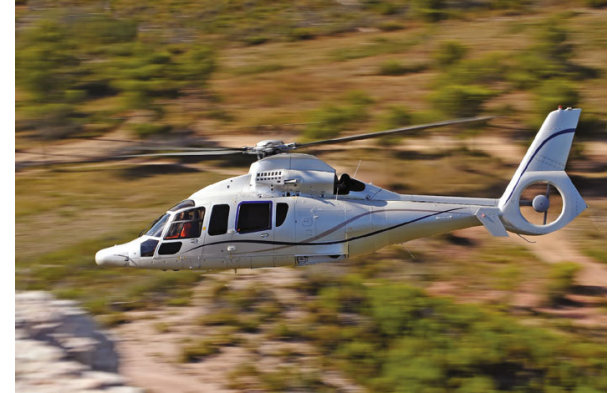

Figure 1: Airbus Helicopter H155 [18].

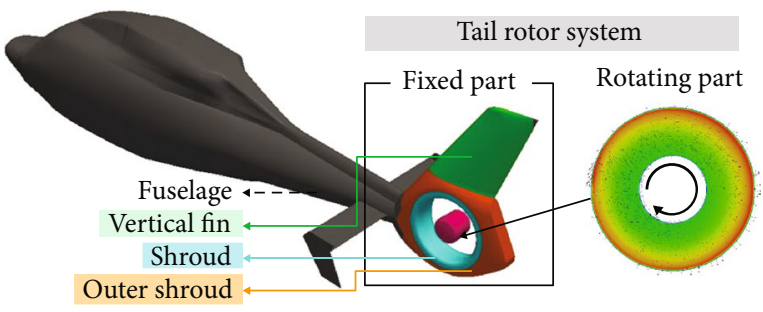

FIgURE 2: Definition of each part of the configuration.

Therefore, the actuator methods provide advantages in evaluating the overall flow characteristics and aerodynamic performance of helicopters under various flight conditions with computational efficiency $[19,20]$.

Recently, Kim et al. [19] developed the improved ASM (IASM), which alleviates the arbitrariness in the selection of the reference line. In the present study, the IASM [19] and general ADM [21] solvers combined with the Rhie-Chow algorithm [22] are used to simulate the main rotor and ducted fan tail rotor, respectively. The IASM is appropriate for analyzing the main rotor because it can simulate the tip vortex and unsteady wake from the rotor blades. The capability of IASM to capture the vortex trajectory has also been verified in a previous study [20]. In addition, the ADM simulates the time-averaged flow of the rotor disk. The prediction capability of ADM for ducted fan tail rotors has been verified by previous studies $[12,16,17]$. In particular, under the condition in which both the main rotor and tail rotor operate simultaneously, the time-scaling problem mentioned previously can be overcome by quasisteady simulation of the tail rotor using the ADM. Figure 3 illustrates a schematic of the helicopter geometry and configuration with rotor modeling.

To implement the ADM and IASM, a source term is added to the momentum equation to incorporate the rotor effects, as defined in Equation (1). The elemental force $(d \vec{F}$ ) in the source term $(\vec{s})$ is determined using the blade element theory as expressed in Equations (2) and (3) for $\mathrm{ADM}$ and IASM, respectively. In these equations, $\alpha_{r}$ represents the angle between the local relative velocity vector and the rotor disk plane, and $N_{b}$ is the number of blades. $f_{\text {Volumeforce }}$ is the volume force shape function that defines the chordwise distribution of the sectional thrust of each blade section. The shape function is normalized to have the maximum value at the quarter chord such that its integration along the chord results in the value of one. $\overrightarrow{n_{N}}$ and $\overrightarrow{n_{T}}$ are unit vectors in the direction normal and tangential to the rotor disk plane, respectively.

$$
\frac{\partial \vec{U}}{\partial t}+(\vec{U} \cdot \nabla) \vec{U}-\nabla \cdot(v \nabla \vec{U})=\vec{s}-\frac{1}{\rho} \nabla p,
$$

where $\vec{s}=d \vec{F} / \rho d V$

$$
d \vec{F}_{\mathrm{ADM}}=\frac{1}{2 \pi r} N_{b} \times \frac{1}{2} \rho U^{2} c\left(C_{l} \cos \alpha_{r}-C_{d} \sin \alpha_{r}\right) \cdot \overrightarrow{n_{N}} d A,
$$

$$
\begin{aligned}
d \vec{F}_{\text {IASM }}= & f_{\text {Volume force }} \frac{1}{2} \rho U^{2}\left\{\left(C_{l} \cos \alpha_{r}-C_{d} \sin \alpha_{r}\right) \cdot \overrightarrow{n_{N}}\right. \\
& \left.+\left(C_{l} \sin \alpha_{r}+C_{d} \cos \alpha_{r}\right) \cdot \overrightarrow{n_{T}}\right\} d A
\end{aligned}
$$

The aerodynamic coefficients $\left(C_{l}, C_{d}\right)$ for the effective angle of attack are obtained from airfoil data tables (C81). In the $\mathrm{ADM}$, the effective angle of attack and corresponding elemental force (Equation (2)) are calculated for each cell in the rotor disk plane by considering the inflow velocity, rotational velocity, and geometric angle of attack at that location. We note that only the thrust component of elemental force (elemental force normal to the disk plane) is considered for the source term in the ADM. In the IASM, the effective angle of attack is determined for each section of a blade by using the correction procedure recommended by Kim et al. [19]. Then, the corresponding sectional force is distributed only to the cells on the blade region along the chordwise direction, according to Equation (3). The momentum source corresponding to the aerodynamic force tangential to the disk plane (torque component) is also imposed in a similar manner. The reader may refer to the references $[19,21]$ for further details.

The IASM and ADM are implemented on an opensource CFD code and OpenFOAM [23]. The PIMPLE [24] algorithm, which combines the pressure implicit with splitting the operators (PISO [25]) and the semi-implicit method for pressure-linked equation (SIMPLE [26]), is used for transient simulations. Since most of the flow field around helicopter rotor blades is substantially incompressible except the region close to the blade surface [27], the incompressible Navier-Stokes algorithm is used. The compressible effects are reflected on sectional aerodynamic performance of the blade via the airfoil data table for $\left(C_{l}, C_{d}\right)$ which is tabulated for several Mach numbers. The airfoil data table used in the present study, which is called the C81 table, is tabulated for angles of attack in the range of $-180^{\circ} \sim 180^{\circ}$ and Mach numbers in the range of $0.3 \sim 0.7$ with an interval of 0.1 . The compressible Reynolds averaged Navier-Stokes simulation is employed to obtain the airfoil data for angles of attack in the range of $-5^{\circ} \sim 30^{\circ}$. The airfoil data beyond this range is obtained by extrapolation following the method described by Leisheman [28]. A second-order backward scheme and second-order Gauss linear upwind scheme are used for 
TABLE 1: Specification of rotors.

\begin{tabular}{lcccccc}
\hline & No. of blades & No. of airfoil & Rotor radius $(\mathrm{m})$ & Solidity & Rotational speed $(\mathrm{rpm})$ & $M_{\text {tip }}$ \\
\hline Main rotor & 5 & 3 (OAF2XX series) & 6.301 & 0.0972 & 342 & 0.66 \\
Tail rotor & 10 & 5 (OAF3XX series) & 0.546 & 0.548 & 3579 \\
\hline
\end{tabular}

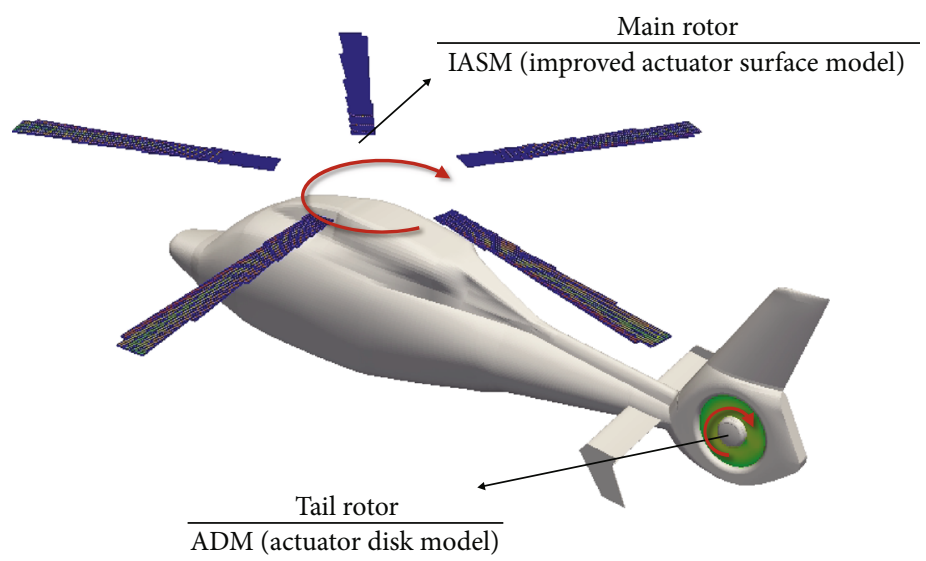

FIGURE 3: Rotating direction and method employed for each rotor.

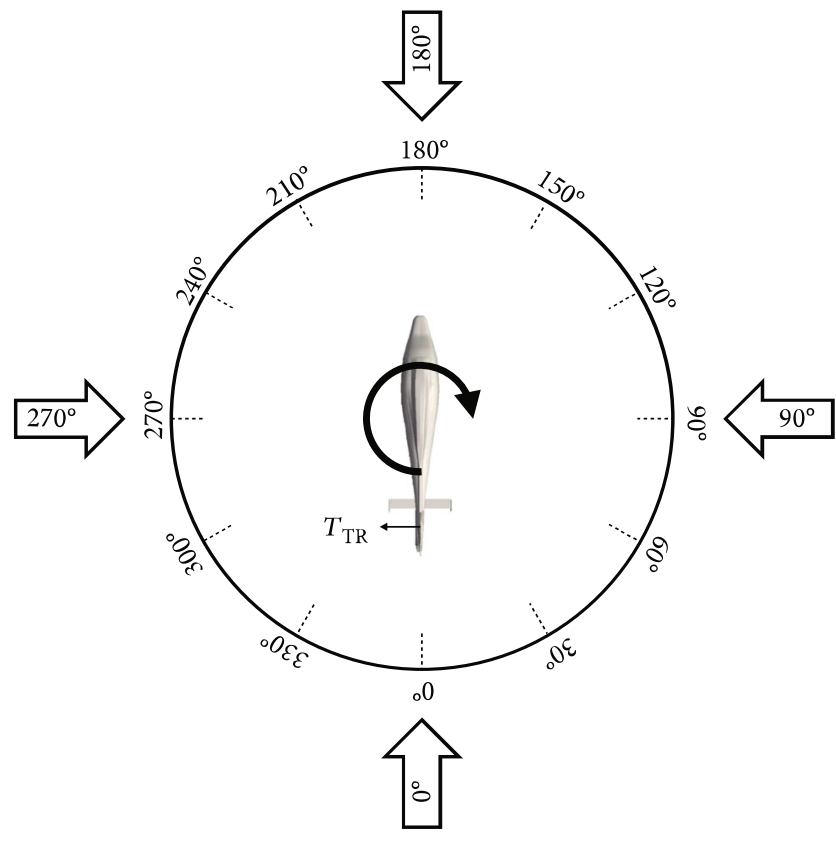

Figure 4: Crosswind directions considered.

temporal discretization and spatial discretization of convective terms, respectively. $k-\omega S S T$, provided by OpenFOAM, is selected as a turbulence model. An $\omega$ wall function described by Menter and Esch [29] is applied for the viscous boundary layer. Spalding's law [30] is used to impart a continuous kinetic turbulence viscosity profile to the wall. The time interval corresponding to $1^{\circ}$ rotation of the main rotor is fixed as the time-step size.

2.3. Computational Grid. A cylindrical computational domain is used to consider the various crosswind directions.
As shown in Figure 5(a), the diameter and height of the cylinder are selected as 80 and 45 times of main rotor diameter, respectively, that of the main rotor diameter. The region around the rotor disk comprises a structured-type grid with hexahedron cells within an individually defined cylindrical region. The ten layers of prism cells are generated over the solid surface. The height of the first grid on the surface is determined such that the target $y+$ is approximately 30 on the fuselage. The entire domain is filled with tetrahedron cells, except for the near rotor region and prism layer. To resolve the flow around the tail rotor sufficiently, a fine mesh is generated with clustering in the vicinity of the helicopter (Figure 5(b)). The total number of computational grids is approximately 19 million.

2.4. Numerical Validation. Preliminary analyses are conducted to validate the grid generation criteria and solver used in the present study. The isolated main rotor and individual ducted fan tail rotor system of H155 are selected and simulated using the IASM and ADM, respectively. For each case, the static aerodynamic performance (thrust or torque) with respect to a collective pitch angle is compared with the reference data from the manufacturer of $\mathrm{H} 155$ and results obtained from individual numerical studies [31, 32] which conducted for similar geometry and condition. Actually, the reference data were shared by the Korea Aerospace Industry (KAI) which obtained the data through the process of joint development and technology transfer program with Airbus Helicopter. However, it is unclear which method is used to obtain those data since AH provided only the data to KAI without further information. It is speculated that the data have been obtained by measurements or by their own design and analysis tools with empirical corrections. 


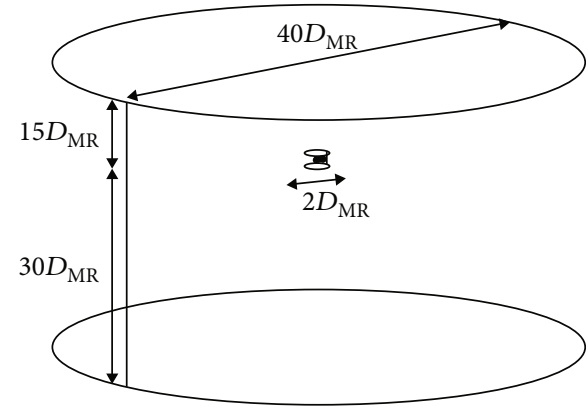

(a) Topology of analyzed domain

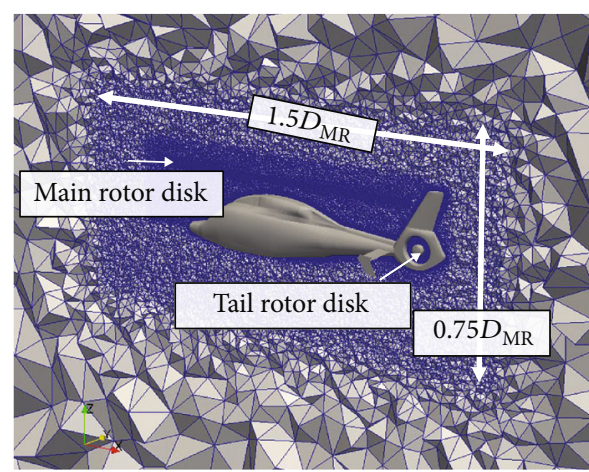

(b) Volume mesh around helicopter

Figure 5: Computational domain.

The performance of the main rotor obtained using IASM is compared with the numerical result of Han [31] using a commercial CFD solver, ANSYS Fluent. In the study of Han [31], a wall-resolved mesh was generated for a blade, and the compressible Navier-Stokes equation was solved based on multiple reference frames with the periodic boundary condition. Figure 6 depicts the comparison of results in terms of the thrust-torque plot. As depicted in the figure, the result of the present study agrees considerably well with that of Han [31]. Because the present IASM provides results close to high-fidelity CFD results, we concluded that it is reasonably accurate in terms of aerodynamic performance.

In the case of the tail rotor, the ducted fan tail rotor system including empennage is considered. The term "empennage" encompasses all the fixed part walls including the isolated ducted fan tail rotor. The results obtained from the $\mathrm{ADM}$ are compared with the numerical results of Woo et al. [32] in Figure 7. Woo et al. used a commercial CFD solver, STAR CCM+, and employed the virtual disk model, which is similar to the ADM of the present study. The total normal force (aligned in the direction of the thrust axis of the tail rotor)-power curves are compared in Figure 7(a). In general, the empennage generates almost half of the total thrust [11]. The thrust sharing between the tail rotor and fixed part (empennage) is also compared in Figure 7(b). The comparison verifies that the ADM of the present study provides an acceptable result in comparison with the reference data. Furthermore, in terms of thrust sharing, the normal force exhibits good agreement for both the tail rotor and empennage. For both main and tail rotor, reference data exhibit the quantitative difference with the numerical results, but the overall tendency is in good agreement. From the comparison with the reference data and the other numerical results, we concluded that the results of the present study are reliable in qualitatively and quantitatively.

In addition, computations for the case of a complete configuration with fuselage in the hovering flight condition are performed as a preliminary analysis. The collective pitch angles of the main rotor and tail rotor are set at $7.5^{\circ}$ and $17^{\circ}$, respectively. The thrust of the main rotor is verified to be almost identical to MTOGW, with an observed difference of less than $0.5 \%$ when all the surface forces acting on the air-

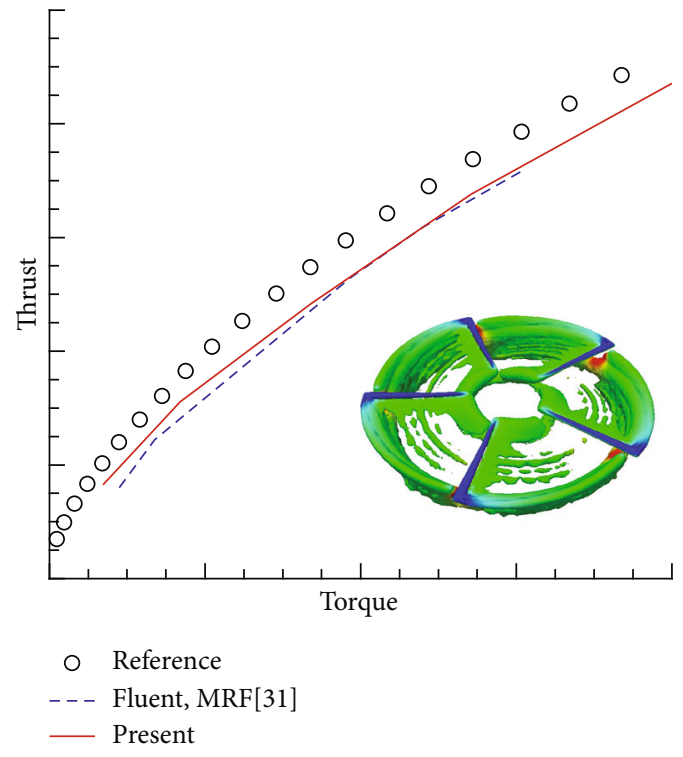

FIgURE 6: Thrust-torque relationship of the main rotor.

frame are considered. Moreover, the thrust of the tail rotor generates torque, which almost completely compensates the torque acting on the whole configuration. The difference is observed to be within $2.5 \%$ of the main rotor torque. The collective pitch angles for the main and tail rotors are fixed at these values in the remainder of the present study.

A grid sensitivity study is carried out using the three levels of grids with different resolutions. To investigate the grid quality in terms of numerical dissipation of wake, grid density is varied mainly in the region around the main/tail rotor. The hovering flight condition is considered, and the calculation time is recorded for 20 revolutions of the main rotor. The total number of cells, calculation time, and results corresponding to the three grids are summarized in Table 2 .

The differences in thrust and torque coefficients are compared with those from the finest grid case (Grid 3). Compared to the case of Grid 3, the Grid 1 and Gird 2 result in $9.8 \%$ and $3.1 \%$ difference in thrust and are 2.6 and 1.7 times computationally more efficient, respectively. In this study, a total of 13 flight conditions are examined with the calculation 


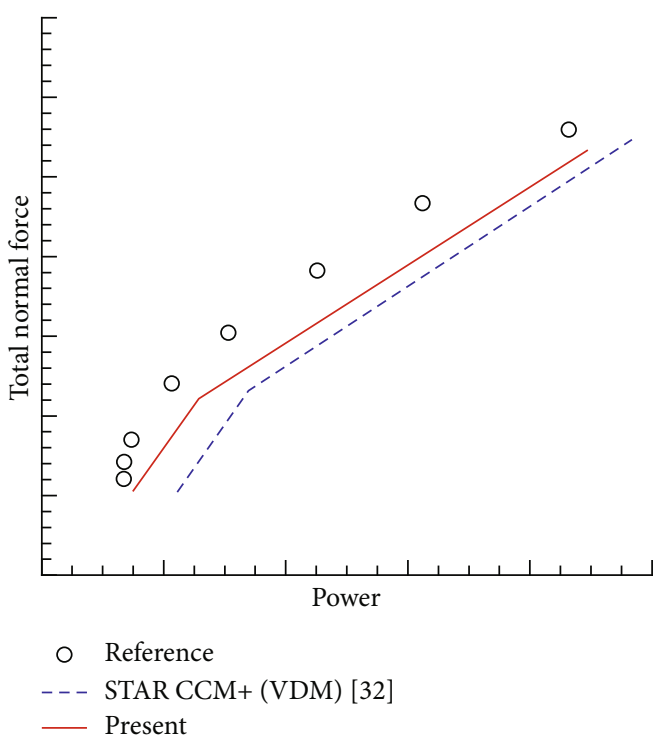

(a) Total normal force-power relation

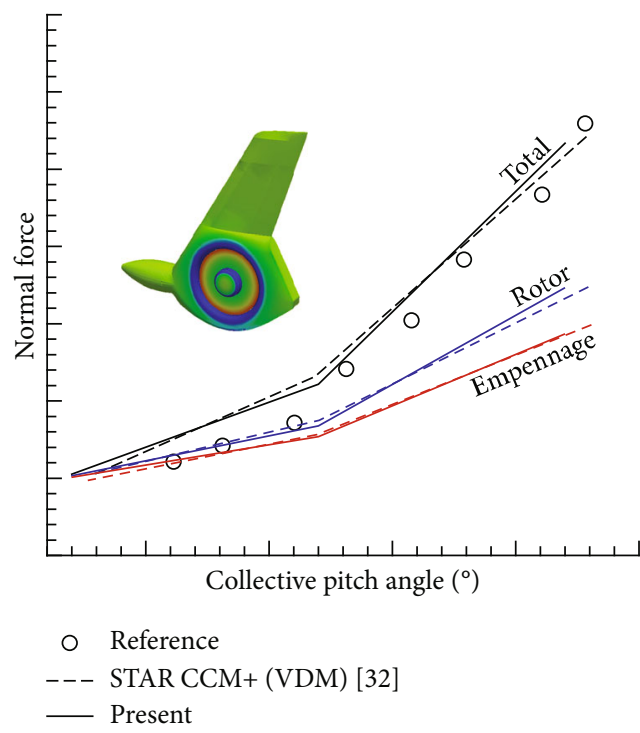

(b) Thrust sharing between rotor and empennage

Figure 7: Aerodynamic performance of tail rotor.

TABLE 2: Information on grid test.

\begin{tabular}{lcccc}
\hline Grid & Total cells & Time $(\mathrm{h})$ & $C_{T, \text { Diff }}(\%)$ & $C_{Q, \text { Diff }}(\%)$ \\
\hline Gird 1 & $13,648,221$ & 42 hours & $9.08 \%$ & $-2.3 \%$ \\
Grid 2 & $19,073,359$ & 63 hours & $3.12 \%$ & $-0.27 \%$ \\
Grid 3 & $26,456,880$ & 108 hours & - & - \\
\hline
\end{tabular}

over the 30 revolutions of the main rotor for several flight conditions. Therefore, to compromise the calculation time and accuracy, Grid 2 is finally chosen and used for all the analysis.

\section{Results and Discussion}

3.1. Flow Characteristics. The case of complete configuration without the main rotor, i.e., the operation of only the tail rotor, is designated as "MR off." For the cases of the main rotor operation under crosswind, each flight condition is denoted by the crosswind angle (e.g., $0^{\circ}$ and $180^{\circ}$ ) for brevity.

3.1.1. Wake Interactions. To identify the general flow features caused by the rotation of the main rotor, the flow fields around the tail rotor at several instances are examined. Figure 8 depicts a series of snapshots at four azimuth angles of the main rotor during a period of the blade's passage. The upper and lower rows represent the Q-criterion colored with the pressure and inflow velocity contour of the tail rotor disk plane, respectively. It is evident from the Q-criteria (upper row) that the main rotor wake hits the vertical fin and flows down toward the outer shroud as the main rotor rotates. Moreover, the inflow velocity contours (lower row) reveal that the wake generated by the main rotor blade is absorbed and immersed in the tail rotor disk. Nevertheless, the inflow velocity of the tail rotor disk does not vary much with the azimuthal location of the main rotor blade, except for marginal differences at a lower side. Further observations indicate that the azimuthal location causes negligible variations in the general flow features under a constant crosswind direction. Hereafter, the snapshot at the instant corresponding to $\psi_{\mathrm{MR}}=0^{\circ}$ is used in the analysis unless stated otherwise.

The crosswind direction affects the main rotor-tail rotor interaction, main rotor-fuselage interaction, and the direction of the wake from each rotor. To evaluate the effect of crosswind on the wake interactions, the $Q$-criteria for several cases of crosswind direction at intervals of $90^{\circ}$ are illustrated in Figure 9. The results for the cases of MR Off and hovering flight with no crosswind (Figures 9(a) and 9(b)) are shown together for comparison. In all the cases, the main rotor produces a complex flow around the tail rotor. When the wind enters from the aft (case of $0^{\circ}$, Figure $9($ c) ), the tail rotor wake is bent toward the forepart. The wake interaction for the cases of $90^{\circ}$ and $270^{\circ}$ is observed to be relatively weak because the wakes from both rotors are in the lateral direction. For the case of $180^{\circ}$, the tail rotor wake convects downward due to the downwash of the main rotor wake. Simultaneously, a spiral pattern is formed because of the periodicity of the main rotor wake.

3.1.2. Main Rotor Effect on Port Wing. The horizontal wing, which is attached to the tail boom, is a rectangular wing with a reverse-camber. The pressure difference between the upper and lower surfaces, the direction of the resulting wing wake, and its vorticity magnitude vary with the crosswind direction. The downwash from the main rotor can, in turn, induce high and low static pressure on the upper and lower surfaces of the wing, respectively. Depending on the crosswind direction, this effect can strengthen the vorticity magnitude of the wing wake. For a specific range of crosswind direction, the 


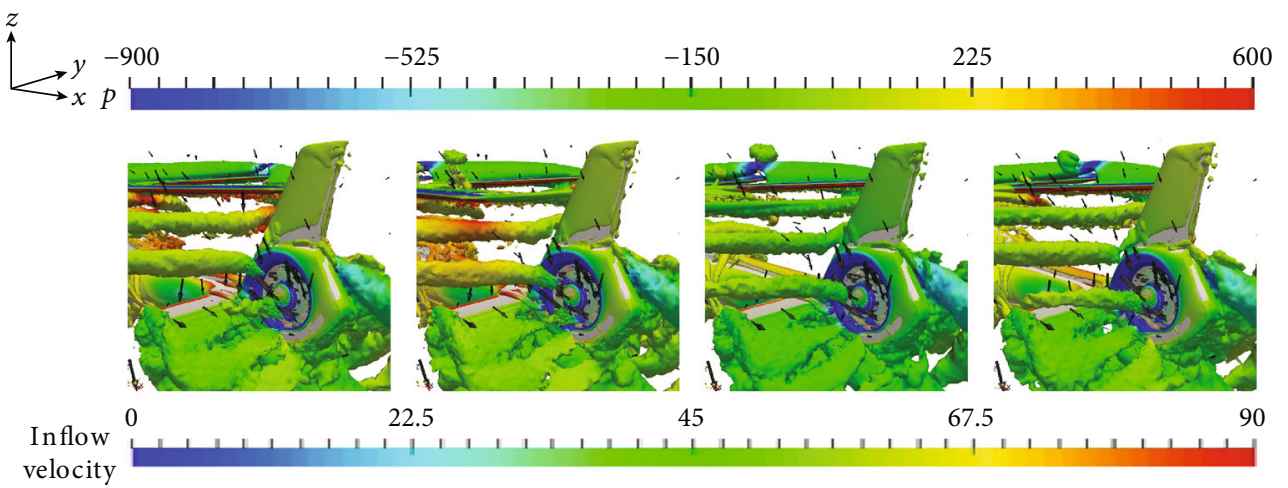

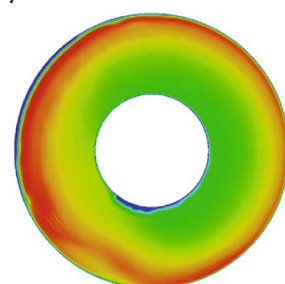

(a) $\psi_{\mathrm{MR}}=0^{\circ}$

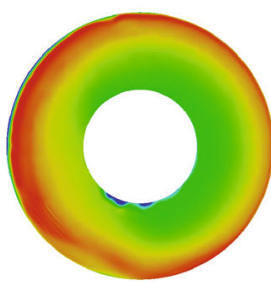

(b) $\psi_{\mathrm{MR}}=18^{\circ}$

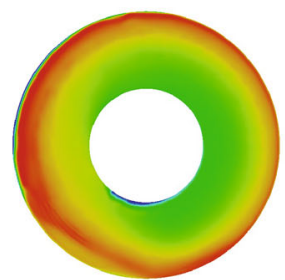

(c) $\psi_{\mathrm{MR}}=36^{\circ}$

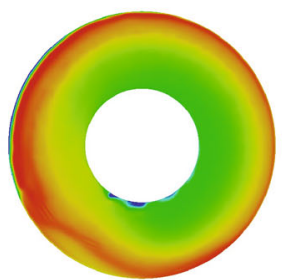

(d) $\psi_{\mathrm{MR}}=54^{\circ}$

FIGURE 8: Q-criteria contour for pressure (upper) and tail rotor disk inflow velocity (lower).

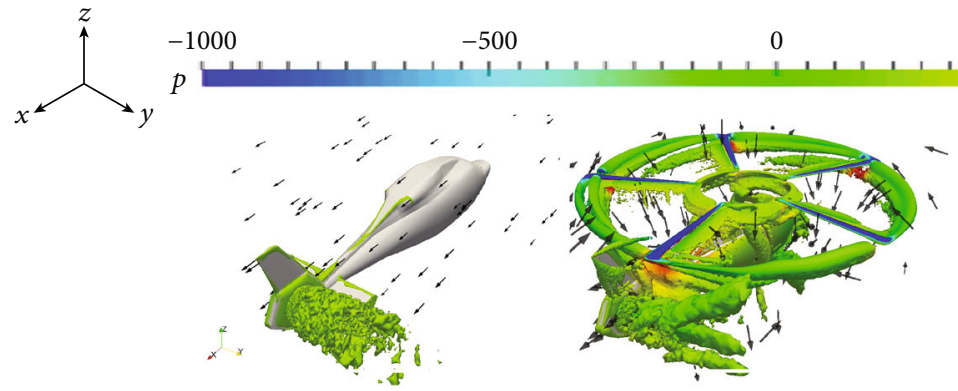

(a) MR Off

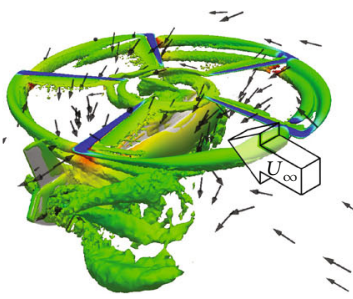

(d) $90^{\circ}$

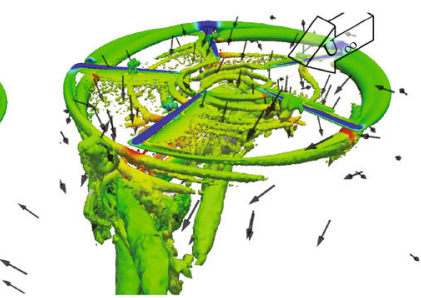

(e) $180^{\circ}$
500,1000

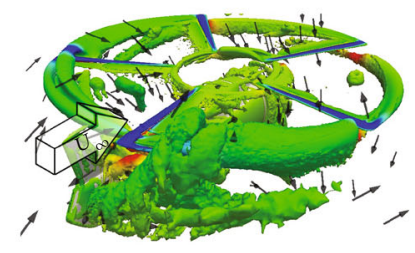

(c) $0^{\circ}$

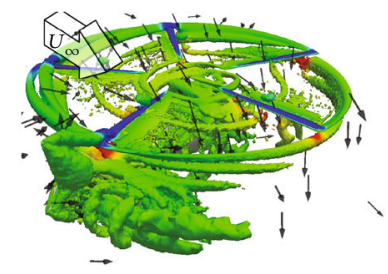

(f) $270^{\circ}$

FIGURE 9: $Q$ iso-surface colored with pressure.

vorticity of the port wing wake can affect the tail rotor disk by entering into the duct inlet.

To estimate the vorticity magnitude of the port wing wake, the time-averaged pressure coefficient on the port wing surface at $y / R_{\mathrm{TR}}=-1.0$ is plotted in Figure 10 for several crosswind direction cases. A comparison of the results reveals that the crosswind direction highly affects the pressure distribution on the port wing surface. For the case of MR Off, the pressure difference is almost zero. The effect of the main rotor wake can also be regarded as insignificant for the case of $0^{\circ}$. Meanwhile, the pressure difference is observed to be significant for the case of $180^{\circ}$, in which the wing generates the largest force in the downward direction. It can be con- cluded that a relatively strong wake is produced from the wing as the wind enters from the forepart.

3.1.3. Tail Rotor Disk Flow. To investigate the influence of the wakes of the main rotor and port wing on the tail rotor, the isovorticity colored with vorticity magnitude in the $y$-direction is illustrated in Figure 11 for the case of $180^{\circ}$. Figure 11 indicates that the wakes of both the main rotor and port wing are absorbed by the tail rotor through suction rather than direct impingement on the tail rotor disk. This is because the fixed part of the tail rotor system prevents their direct impingement. In particular, the two strongest main tip vortices (designated as blade wakes) collide with the outer shroud. 


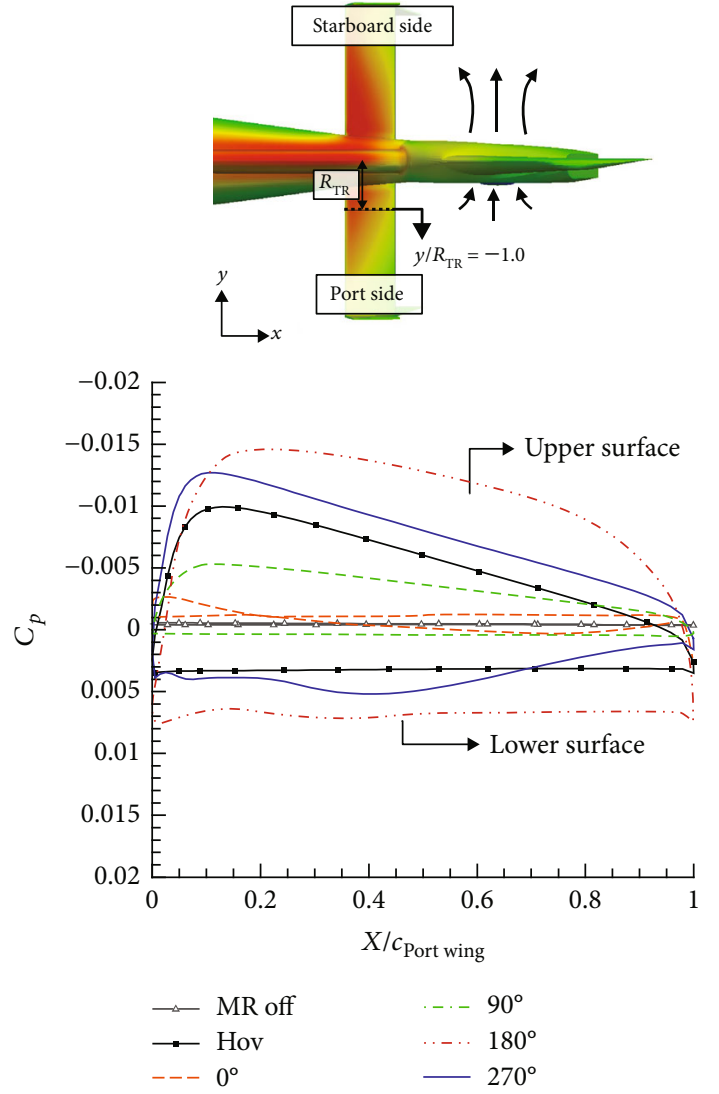

FIGURE 10: Time-averaged pressure coefficient on the port wing at $\mathrm{y} / R_{\mathrm{TR}}=-1.0$.

The tip vortices generated from the main rotor blades rotate about the negative $y$-axis. This rotational direction of main rotor wake is counterclockwise from the figure's perspective and is opposite to that of the tail rotor. Meanwhile, the port wing wake contains a vorticity component whose direction is the same as that of the tail rotor. The rotational directions of the rotors, main rotor tip vortices, and wing wake are depicted in Figure 12.

Although the main rotor tip vortices are weakened after colliding with the fixed part, they still appear to considerably influence the tail rotor performance. Figure 13 depicts an instantaneous inflow contour on the tail rotor disk plane colored with velocity magnitude in the hovering flight condition. For illustration purposes, a few velocity vectors in the disk plane are plotted together. A detailed investigation of the results reveals that the inflow on the tail rotor can be divided into the main rotor-wake and port wing-wake dominated regions. To identify their effect more clearly, the contour for the case of MR off is compared. The arrows with the solid line and dotted line over the contour indicate the flow direction opposite and same to the tail rotor rotation, respectively. In the case of hovering flight, the effect of the main rotor wake encompasses the entire region of the upper half and the region marginally below the hub. Moreover, the magnitude of in-plane velocity owing to the main rotor wake is relatively large.
Therefore, the velocity induced by the main rotor wake significantly influences the tail rotor. Meanwhile, the port wing wake affects most of the lower half of the rotor disk. The magnitude of the in-plane velocity is relatively weak. The port wing wake induces flow velocity in the direction of and opposite to the tail rotor rotation, around the tip (dotted-line arrow) and hub (solid-line arrow), respectively. Furthermore, the additionally induced velocities cause a variation in the relative velocity, i.e., the effective angle of attack determining the local $C_{l}$ and $C_{d}$ of the cells on the rotor disk.

The circumferential velocity in the counterclockwise direction along the vertical centerline of the disk plane is plotted in Figure 14. The deviation in the circumferential velocity from the case of MR off can be regarded as a consequence of the wakes, as discussed above. In most of the upper half $\left(\mathrm{z} / r_{\mathrm{TR}}>0.4\right)$ and the portion of the lower half adjacent to the hub $\left(-0.7<\mathrm{z} / r_{\mathrm{TR}}<-0.4\right)$, the circumferential velocity is increased owing to the main rotor wake. An increase in the circumferential velocity causes an increase in the effective angle of attack for local cells on the disk plane. Therefore, it can be concluded that the main rotor wake causes an increase in the tail rotor thrust. This can be verified from the inflow contour, in which the apparent increase in the inflow magnitude around the upper tip region is observed. Meanwhile, in the remainder of the lower half around the tip $\left(\mathrm{z} / \mathrm{r}_{\mathrm{TR}}<-0.7\right)$, the circumferential velocity decreases due to the port wing wake.

3.1.4. Effects of Crosswind Direction. The effects of crosswind direction on the tail rotor are analyzed. Figure 15 illustrates snapshots with $Q$-criterion around the tail rotor system for the cases of four crosswind directions at intervals of $90^{\circ}$. When the flow enters from the aft $\left(0^{\circ}\right.$, Figure 15(a)), the main rotor wake rarely reaches the tail rotor disk. The port wing generates wake in the forward direction. This wake is convected toward the starboard side rather than entering the tail rotor disk. Therefore, the variation in the inflow contour on the disk plane is almost negligible for this case. In contrast, for the case of $180^{\circ}$, the tail rotor is considerably affected by the main rotor and port wing wakes. In the cases of sideward flow $\left(90^{\circ}\right.$ and $\left.270^{\circ}\right)$, the observed influence is weaker than that in the case of $180^{\circ}$.

Figure 16 depicts a series of inflow contours on the tail rotor disk with in-plane velocity vectors $\left(0^{\circ}\right.$ and $90^{\circ} \sim$ $270^{\circ}$ at intervals of $30^{\circ}$ ). As the crosswind flows from the forepart $\left(180^{\circ}\right)$, the strength and influential region of the main rotor wake increase. Simultaneously, the area of the port wing wake influence is suppressed, although the strength is increased (Figure 10). The impact of the main rotor wake increases gradually as the crosswind direction varies from $90^{\circ}$ to $180^{\circ}$. It decreases again as the crosswind direction increases further to $270^{\circ}$. The effect of the main rotor wake appears to be stronger when the crosswind enters from the duct inlet (Figure 16(f), case of $210^{\circ}$ ) than when it enters from the duct outlet (Figure 16(d), case of $150^{\circ}$ ).

Previous studies on open-type tail rotors $[1,4-7,32]$ have identified that the case of $90^{\circ}$ corresponds to a 


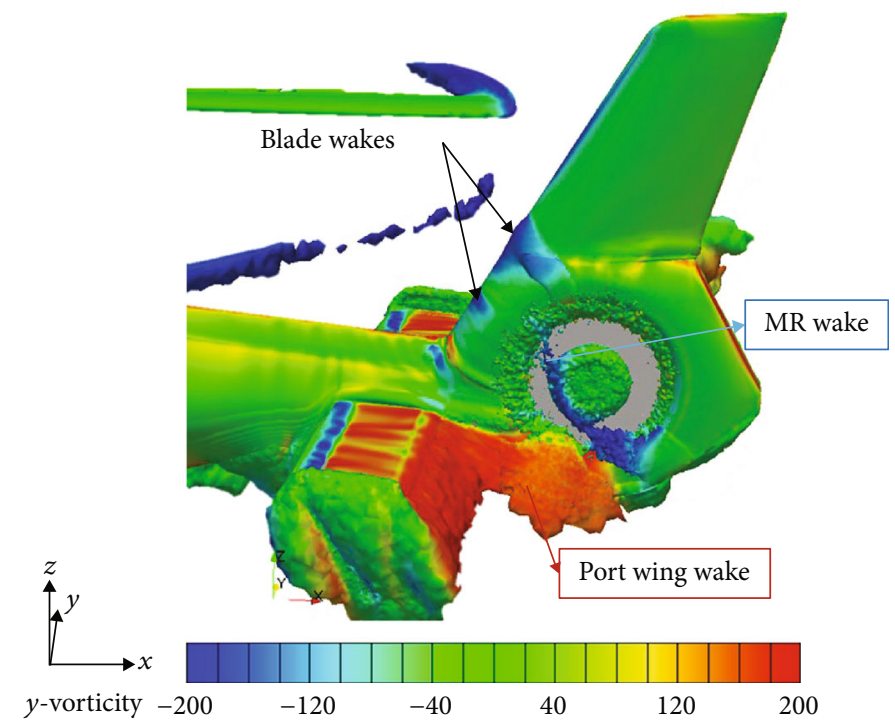

FIGURE 11: Isovorticity contour with $y$-vorticity for $180^{\circ}$.

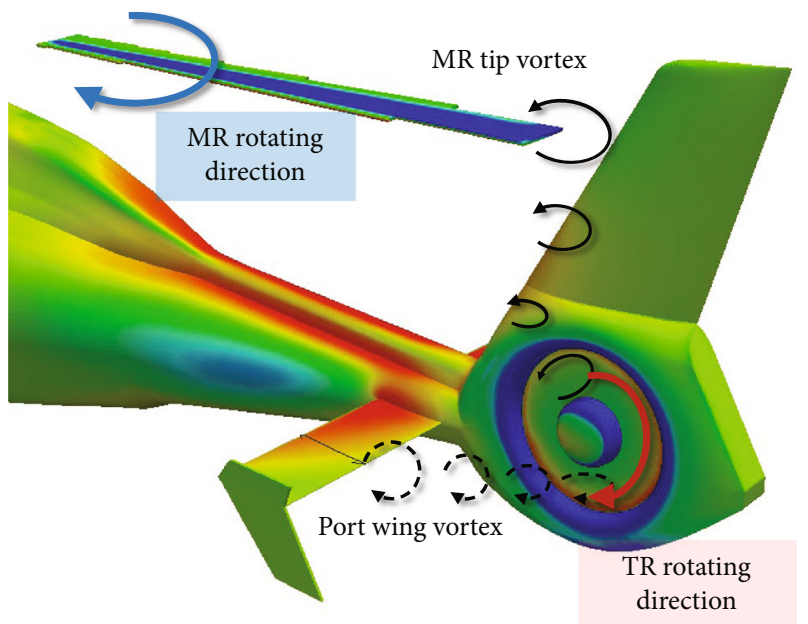

FIGURE 12: Schematic of vortex and wake directions around the tail rotor.

hazardous condition because of the likelihood of occurrence of a vortex ring state. However, for the ducted fan tail rotor, the vortex ring state does not appear to occur in this condition (Figures $9(\mathrm{~d})$ and 16(b)). Instead, the generation of the tip vortex is suppressed considerably, and the flow tends to expand in conjunction with the shroud [13]. Moreover, when the crosswind direction is opposed to the direction of the tail rotor wake, the tail rotor wake turns around the outer geometry of the tail rotor system without returning to the duct.

\subsection{Aerodynamic Forces}

3.2.1. Averaged Forces. Figure 17 depicts the time-averaged thrust with respect to crosswind direction. The root mean square (RMS) of the thrust fluctuation for each flight condition which is defined as Equation (4) is indicated as the error bar. All the results are normalized by the average thrust for the case of the hovering condition.

$$
T_{\mathrm{RMS}}(\%)=\sqrt{\frac{1}{N} \sum_{n=1}^{N}\left(\frac{T_{\mathrm{TR}}-T_{\mathrm{TR}, \mathrm{avg}}}{T_{\mathrm{TR}, \text { hov,avg }}}\right)^{2}} \times 100 .
$$

As discussed previously, the thrust of the tail rotor mainly depends on the interaction with the main rotor and port wing wakes, which causes an increase and a decrease in the thrust, respectively. The effects are observed to be dominant when the wind flows from the forepart $\left(180^{\circ}\right)$, and the least significant when the wind flows from the aft part $\left(0^{\circ}\right)$. Consequently, the variation in the tail rotor thrust is observed to vary from $-17.5 \%$ (at $330^{\circ}$ ) to $12.1 \%$ (at $180^{\circ}$ ). The RMS amplitude is observed to be less than $1.4 \%\left(0^{\circ}, 90^{\circ}, 270^{\circ}\right)$ and $1.9 \%\left(180^{\circ}\right)$. That is, the RMS amplitude is the largest for the case of $180^{\circ}$.

Figure 18 compares the normal force from an individual component of the tail rotor system, in the direction of the tail rotor thrust. Again, the forces are normalized by the total force of the tail rotor system in the hovering condition. With regard to the fixed parts, the forces generated by the outer shroud and vertical fin exhibit an apparent sinusoidal pattern with respect to the crosswind direction (odd function about $\left.180^{\circ}\right)$. This can be attributed to the fact that the outer shroud and vertical fin are directly exposed to the external flow (crosswind), and its influence is the dominant determinant of the variation in the resulting force. Meanwhile, the force from the tail rotor exhibits almost symmetric about $180^{\circ}$ as a result of the interactions discussed previously. The total normal force of the tail rotor system is the sum of the contributions of all the components. The effect of crosswind direction on the total normal force in terms of the maximum deviation from the hovering condition is observed to be more pronounced for the range between $180^{\circ}$ and $360^{\circ}$. 

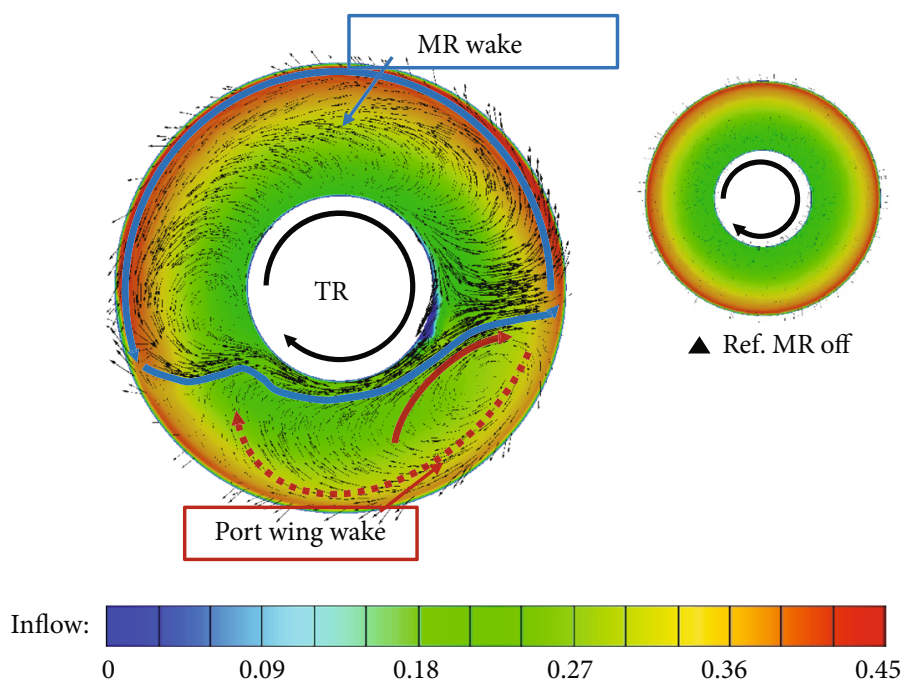

FIGURE 13: Inflow contour with in-plane velocity vector in hovering flight.

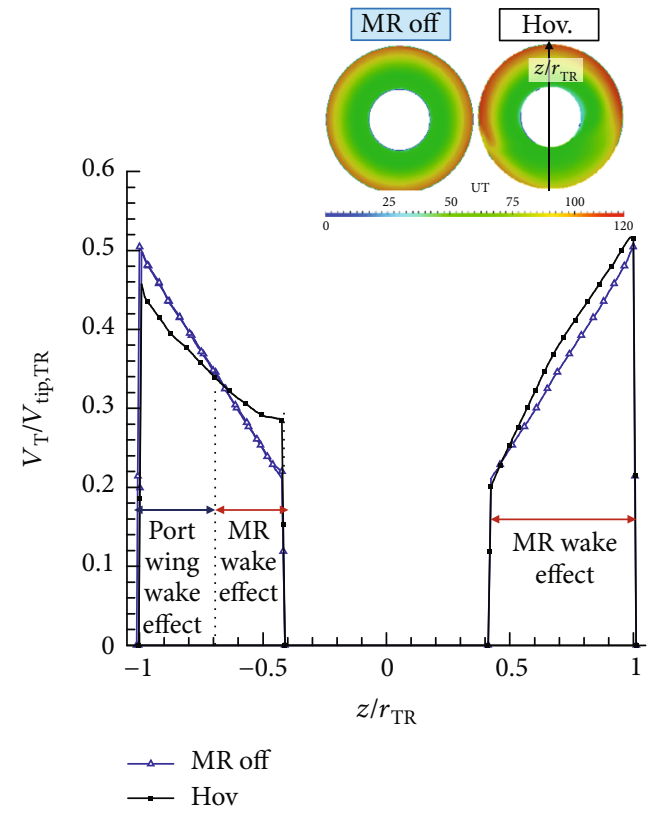

FIGURE 14: Circumferential velocity along the vertical centerline.

To evaluate the characteristics of directional stability, the variation of the yawing moment relative to the hovering condition is depicted in Figure 19. The center of the main rotor is set as the moment reference point. The moments are normalized by the main-rotor torque in the hovering condition. The directional stability is assessed by evaluating the gradient of total yawing moment. It is directionally stable when the helicopter tends to rotate in the same direction with incoming crosswind direction. For the configuration of the present analysis, it ensures directional stability when the total yawing moment is increased as the crosswind direction is changed from $0^{\circ}$ to $360^{\circ}$. The pedal position of the tail rotor is evaluated to assess the controllability. The required collective pitch angle is determined from the performance of the isolated ducted fan tail rotor (Figure 7) without considering the influence of the flow interaction and thrust variation on each component, with respect to the crosswind direction. The pedal margin, which is the remaining space for manipulating the collective pitch angle of the tail rotor from the full left and full right pedals, is also identified based on the pedal position.

Figure 19 shows that the tail rotor system contributes to most of the variations in the total yawing moment. As anticipated, the variation is significant for crosswind of $180^{\circ} \sim$ $360^{\circ}$ because the variation in the $F_{y}$ of the tail rotor system is more pronounced in this range (see Figure 18). The fuselage, which is also under the influence of the main-rotor wake, contributes less to the variation in the yawing moment. The variation in the total yawing moment ranges from $-27 \%$ (at $60^{\circ}$ ) to $33 \%\left(\right.$ at $270^{\circ}$ ) of the main-rotor torque in the hovering condition. The helicopter is directionally stable in the $150^{\circ} \sim 270^{\circ}$, as marked by shading area. The pedal position for achieving directional stability is observed to vary from 53.5\% (lowest case) to $67.6 \%$ (highest case). The corresponding pedal margins to the full left and full right are $53.5 \%$ and $32.3 \%$, respectively. From these results, even the yawing moment varies with crosswind direction, the pilot has enough control margin for the current model of helicopter.

3.3. Comparison with Open-Type Tail Rotor. Figure 20 illustrates the schematics of flow around both the ducted fan tail rotor and open-type tail rotor. The left and right figures show rotor inlet-view and top-view, respectively. The right figures compare the effect of crosswind when the flow is coming from $90^{\circ}$, where the vortex ring state could be induced.

For the ducted fan tail rotor, direct impingements of the main rotor and port wing wakes are prevented because the rotor is protected by the fixed parts of the tail rotor system. The phenomenon of reentry of the tip vortex, which is frequently encountered in the open-type tail rotor, is also prevented. The thrust of the open-type tail rotor can be decreased by a maximum of $80 \%[1,24,33]$, whereas the 


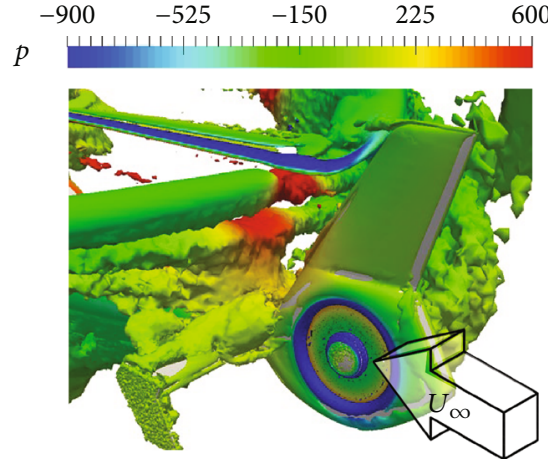

(a) $0^{\circ}$

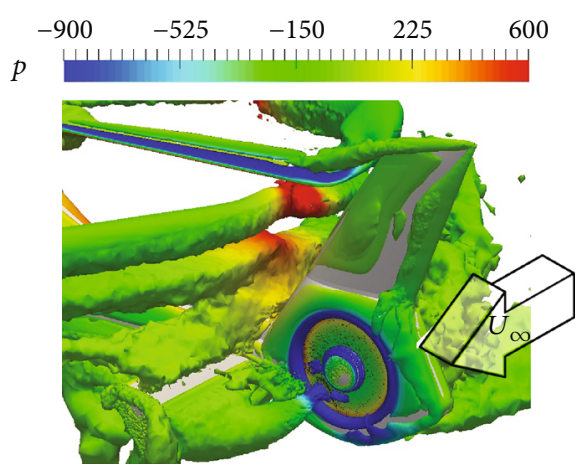

(b) $90^{\circ}$

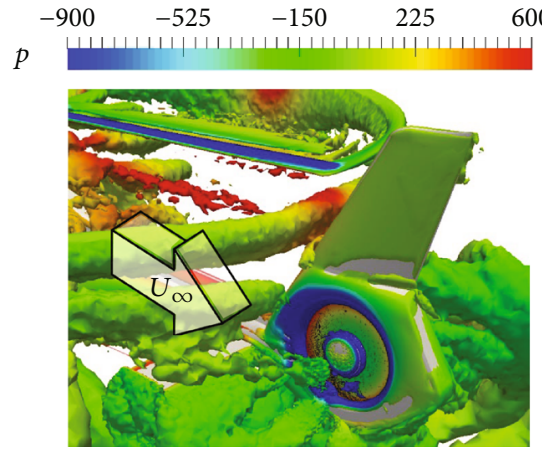

(c) $180^{\circ}$

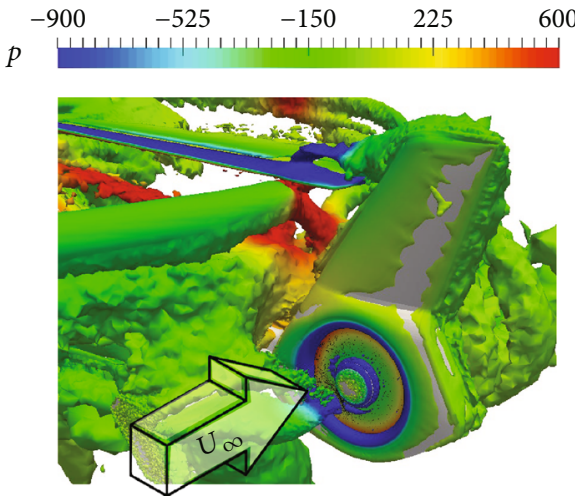

(d) $270^{\circ}$

FIGURE 15: Q-criterion around the tail rotor.

Inflow

velocity

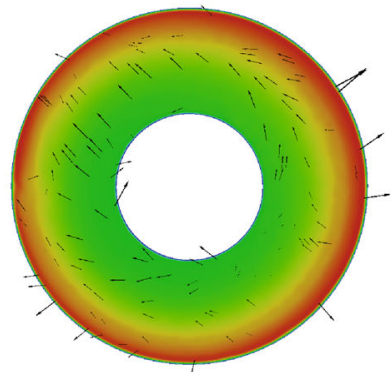

(a) $0^{\circ}$

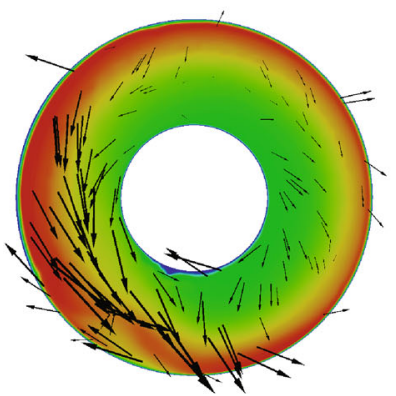

(e) $180^{\circ}$

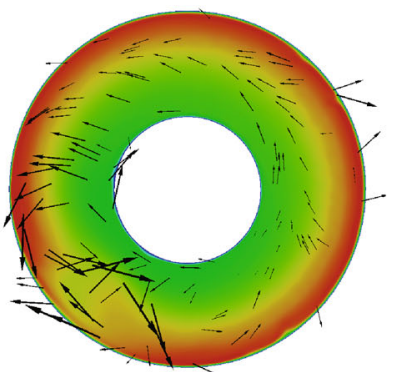

(b) $90^{\circ}$

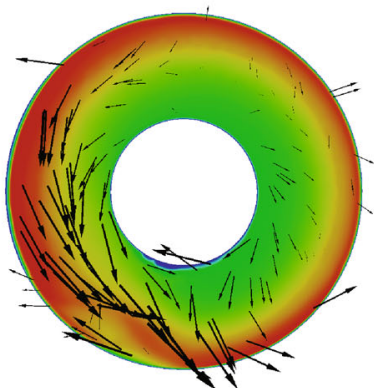

(f) $210^{\circ}$

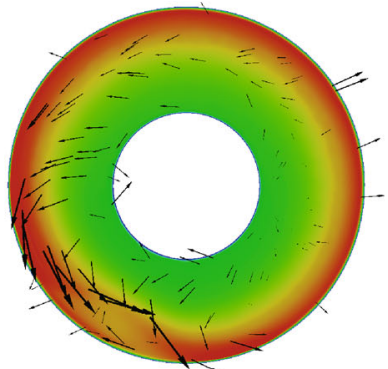

(c) $120^{\circ}$

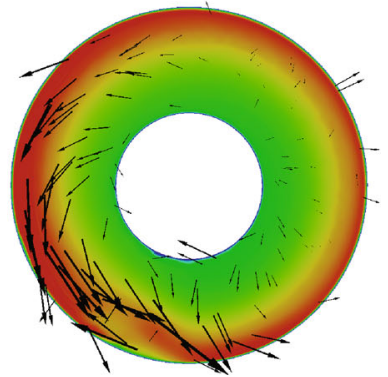

(g) $240^{\circ}$

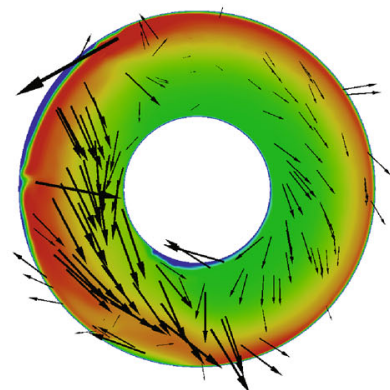

(d) $150^{\circ}$

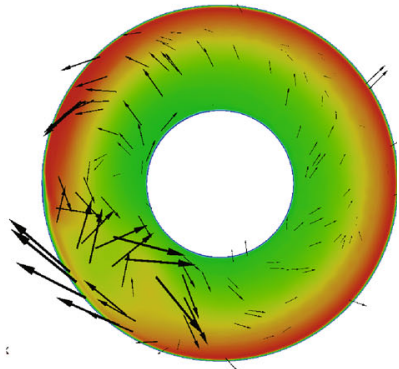

(h) $270^{\circ}$

Figure 16: Inflow contour with velocity vector with respect to crosswind angle. 


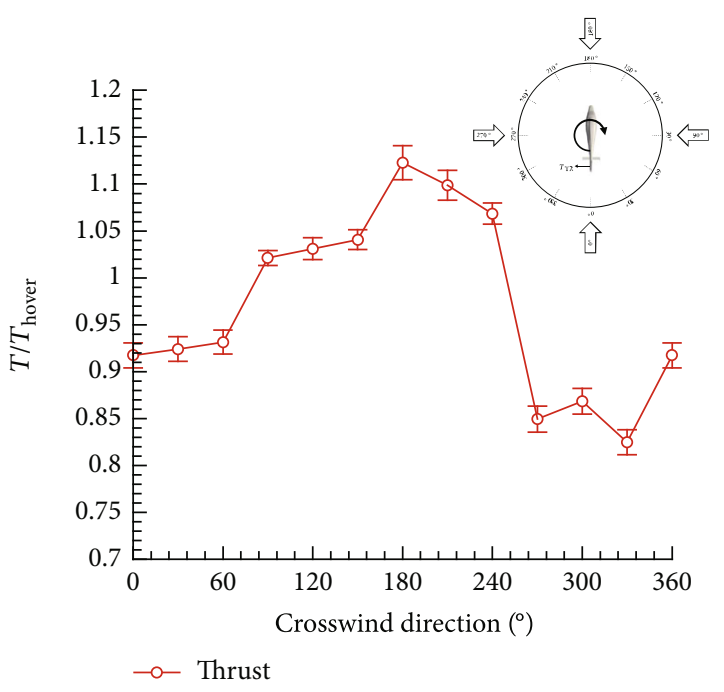

Figure 17: Time-averaged thrust of the tail rotor.

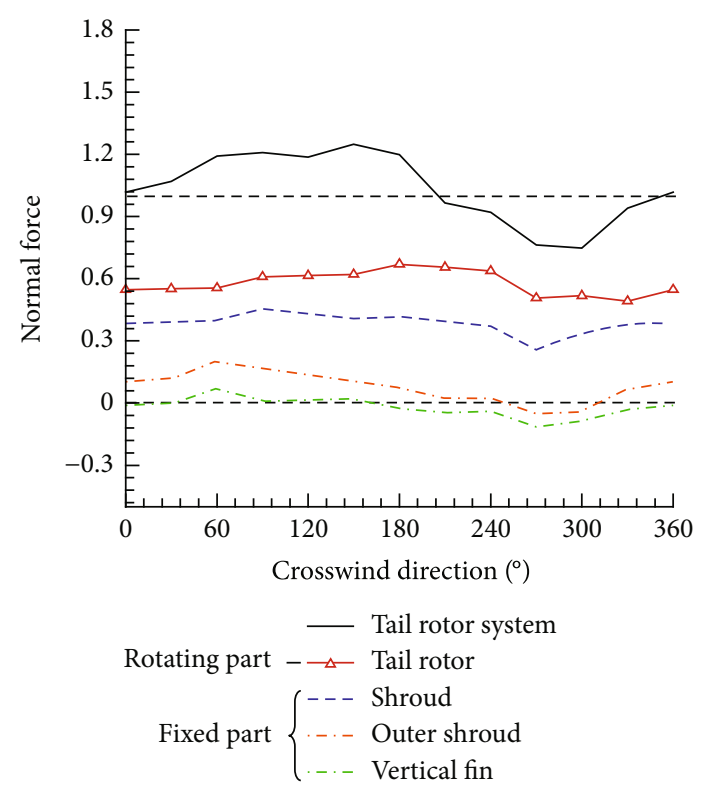

FIGURE 18: Components of normal force of the tail rotor system.

thrust of the ducted fan tail rotor is reduced by a maximum of $10 \%$ (for the case of $330^{\circ}$ ). The maximum RMS amplitude of the thrust fluctuation is observed to be approximately $2.1 \%$. Meanwhile, for open-type tail rotors, it is observed to be larger than 25\% (SH-2 [34]) and 20\% (YAH-64 [35]), under 20 knots at a certain crosswind direction. In terms of the total yawing moment, the ducted fan tail rotor exhibits a higher degree of pedal margin relative to the open-type tail rotor. The pedal margin of the ducted fan tail rotor is $53.5 \%$ and $32.3 \%$ to the full left and full right, respectively. For the open-type tail rotor, $\mathrm{SH}-2[34]$ and Lynx $[6,7]$ present 24$40 \%$ and $10-40 \%$ of the pedal margin, respectively. Thus, it can be concluded that the ducted fan tail rotor system can suppress the severe degradation and oscillation of the thrust apparent in the open-type tail rotor.

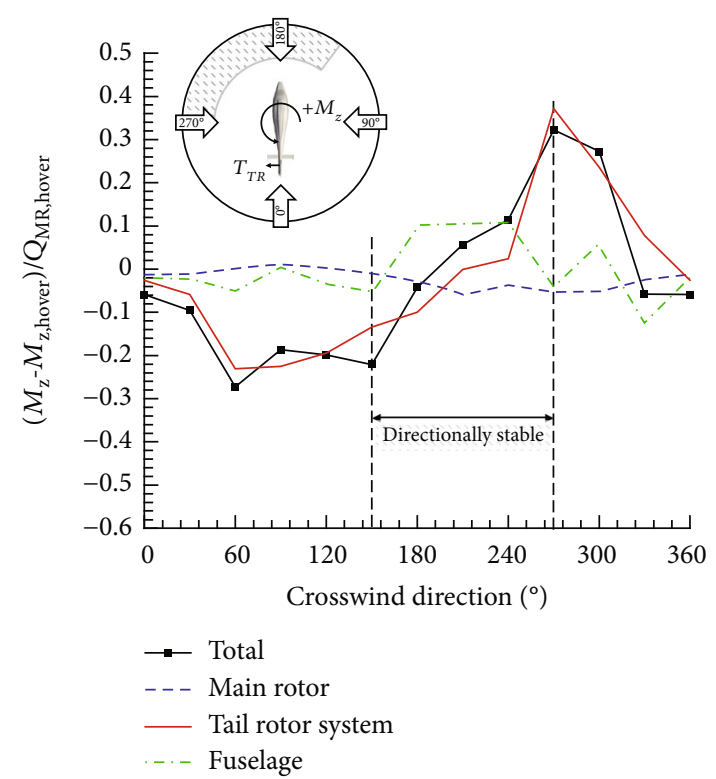

Figure 19: Yawing moment of each component.

\section{Conclusions}

A numerical analysis has been conducted for the complete configuration of a helicopter with a ducted fan tail rotor. The hovering condition with various crosswind directions was studied to investigate the flow characteristics at lowspeed crosswind. For a time- and cost-efficient analysis, the IASM and ADM were applied to the main rotor and tail rotor, respectively. The variations in the averaged and fluctuation forces with respect to the crosswind direction were evaluated, as well as the flow characteristics. Additionally, the aerodynamic performances were compared with the results from previous researches that analyzed the open-type tail rotor. The quantitative accuracy of aerodynamic performance could vary depending on various factors such as the grid resolution, numerical scheme, and numerical methods employed. Nevertheless, the qualitative trends of aerodynamic forces and the characteristics of flow-field investigated in this study seem to be useful as basic references for the initial design of rotorcrafts with similar concepts.

The results revealed that

(1) The performance and flow characteristics of the tail rotor are influenced substantially by the wakes of the main rotor and port wing. The direction of the wakes is directly affected by the wind direction. The effects of the main rotor and port wing wake on the tail rotor when the flow is from the fore $\left(180^{\circ}\right)$ are considerably high

(2) The main rotor tip vortex rotates in the direction opposite to the tail rotor and contributes to an increase in the circumferential velocity on the tail rotor disk plane. This eventually causes an increase in the tail rotor thrust. The contribution of the port 

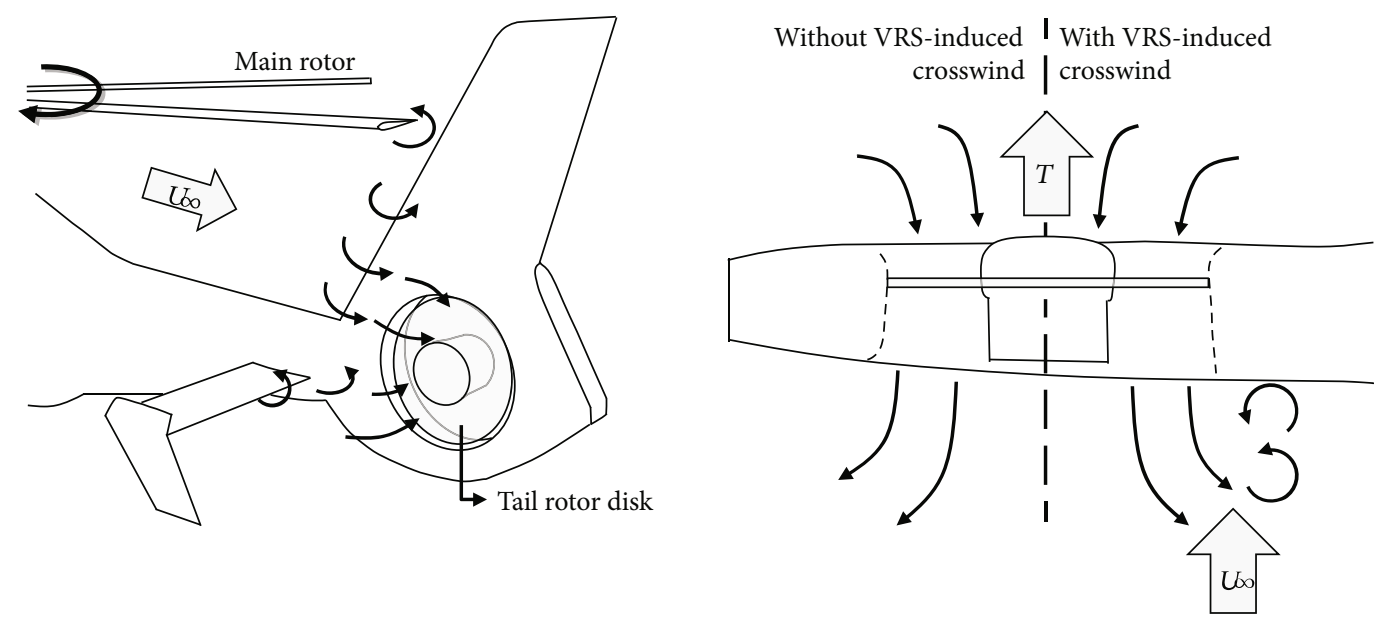

(a) Ducted fan tail rotor

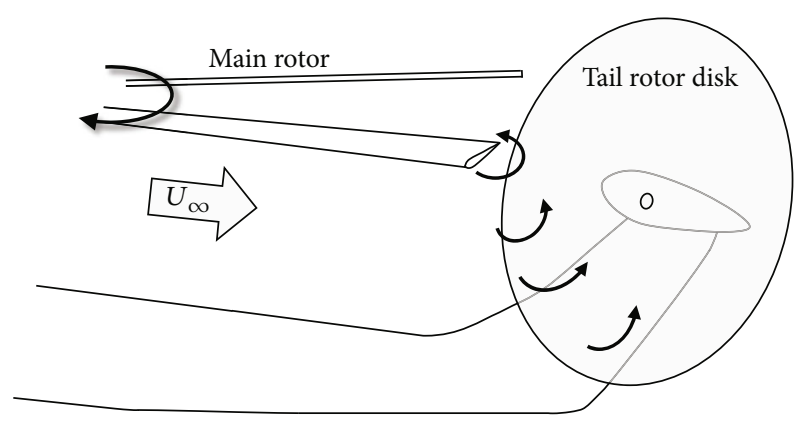

(b) Open-type tail rotor
Without VRS-induced I With VRS-induced crosswind | crosswind

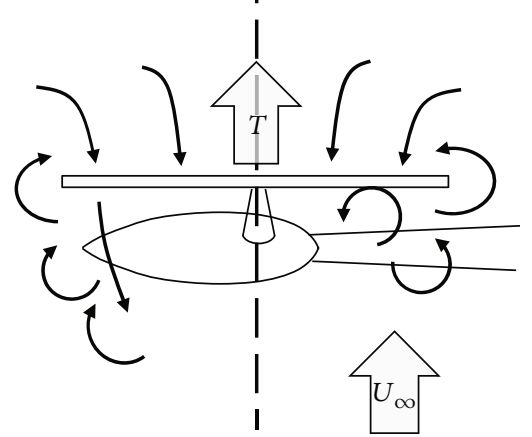

FIgURE 20: Schematics of flow around the tail rotor.

wing wake is observed to be opposite to that of the main rotor tip vortex

(3) The tail rotor thrust is maximum when the wind enters from the fore (case of $180^{\circ}$ ). This is because the main rotor wake is the most influential factor for increasing the tail rotor thrust under this condition. Meanwhile, the substantial influence of the main rotor wake induces adverse effects in terms of the fluctuation in the tail rotor thrust

(4) The tail rotor system contributes to most of the variations in the total yawing moment. The variation in the yawing moment exhibits an asymmetric tendency with respect to $180^{\circ}$ because of the forces of the tail rotor system. The total yawing moment attains its maximum and minimum values in the case of $270^{\circ}$ and $60^{\circ}$, respectively

(5) In terms of aerodynamic performances, the helicopter with a ducted fan tail rotor is observed to have higher stability for various crosswind directions than that with an open-type tail rotor. This can be attributed to the geometrical characteristics of the ducted fan. Regardless of the wind direction, the direct influence of the disturbed wakes does not occur because the rotor is protected by the fixed part of the tail rotor system. Therefore, only the weakened wake and vortex are absorbed and immersed in the tail-rotor disk by the suction

\section{Data Availability}

The data used to support the observations of this study are available from the corresponding author upon request.

\section{Conflicts of Interest}

The authors declare that there is no conflict of interest with regard to the publication of this paper.

\section{Acknowledgments}

This work was conducted at High-Speed Compound Unmanned Rotorcraft (HCUR) research laboratory with the support of the Agency for Defense Development (ADD). This research was supported by the Study on the Core Technologies of Electric Vertical Take-Off \& Landing Aircraft (FR20A04) through the National Research Council of Science and Technology. 


\section{References}

[1] W. Wiesner and G. Kohler, "Tail rotor performance in presence of main rotor, ground, and winds," Journal of the American Helicopter Society, vol. 19, no. 3, pp. 2-9, 1974.

[2] W. White, "Unanticipated right yaw in helicopters," FAA Advisory Circular, no. 90-95, 1995.

[3] D. C. Dugan and K. J. Delamer, The implications of handling qualities in civil helicopter accidents involving hover and low speed flight, NASA Technical Memorandum, 2005.

[4] K. B. Amer and A. Gessow, Charts for Estimating Tail-Rotor Contribution to Helicopter Directional Stability and Control in Low-Speed Flight, NACA Technical Report, 1955.

[5] D. Snellen, OH-58 loss of tail rotor effectiveness, US Army Aviation Digest, 1984.

[6] A. Ellin, "An inflight investigation of LYNX AH MK5 main rotor/tail rotor interactions," in 19th European Rotorcraft Forum, Cernobbio, Italy, September 1993.

[7] A. Ellin, "Lynx main rotor/tail rotor interactions: mechanisms and modelling," Proceedings of the Institution of Mechanical Engineers, Part G: Journal of Aerospace Engineering, vol. 208, no. 2, pp. 115-128, 1994.

[8] J. Thiemeier, C. Öhrle, F. Frey, M. Keßler, and E. Krämer, "Aerodynamics and flight mechanics analysis of Airbus Helicopters' compound helicopter RACER in hover under crosswind conditions," CEAS Aeronautical Journal, vol. 11, no. 1, pp. 49-66, 2020.

[9] R. Mouille, "The "Fenestron," shrouded tail rotor of the SA. 341 Gazelle," Journal of the American Helicopter Society, vol. 15, no. 4, pp. 31-37, 1970.

[10] A. Vuillet and F. Morelli, "New aerodynamic design of the Fenestron for improved performance," in 12th European Rotorcraft Forum Proceeding, Garmisch-Partenkirchen, Germany, September 1986.

[11] B. N. Bourtsev and S. V. Selemenev, "Fan-in-fin performance at hover computational method," in 26th European Rotorcraft Forum Proceeding, The Hague, Netherlands, September 2000.

[12] Y. Cao and Z. Yu, "Numerical simulation of turbulent flow around helicopter ducted tail rotor," Aerospace Science and Technology, vol. 9, no. 4, pp. 300-306, 2005.

[13] R. G. Rajagopalan and C. N. Keys, "Detailed aerodynamic analysis of the RAH-66 FANTAIL ${ }^{\mathrm{TM}}$ using CFD," Journal of the American Helicopter Society, vol. 42, no. 4, pp. 310-320, 1997.

[14] M. Kainz, F. Danner, H.-P. Kau, and F. Le Chuiton, "Numerical investigation into the unsteady aerodynamics of a ducted helicopter tail rotor under side-wind conditions," in ASME Turbo Expo 2010: Power for land, Sea, and Air, pp. 87-98, Glasow, Scotlnad, UK, June 2010.

[15] M. Marino, N. Gourdain, J.-F. Boussuge, G. Legras, and D. Alfano, "A Large Eddy Simulation of the Fenestron ${ }^{\circledR}$ at high blade pitch angle," in 42th European Rotorcraft Forum Proceeding, Lille, France, September 2016.

[16] E. Alpman, L. N. Long, and B. D. Kothmann, "Understanding ducted rotor antitorque and directional control characteristics part I: steady state simulation," Journal of Aircraft, vol. 41, no. 5, pp. 1042-1053, 2004.

[17] E. Alpman, L. N. Long, and B. D. Kothman, "Understanding ducted rotor antitorque and directional control characteristics part II: unsteady simulations," Journal of Aircraft, vol. 41, no. 6 , pp. 1370-1378, 2004.
[18] https://www.airbus.com/helicopters/civil-helicopters/ medium/h155.html.

[19] T. Kim, S. Oh, and K. Yee, "Improved actuator surface method for wind turbine application," Renewable Energy, vol. 76, pp. 16-26, 2015.

[20] D. Linton, G. Barakos, R. Widjaja, and B. Thornber, "Coupling of an unsteady aerodynamics model with a computational fluid dynamics solver," AIAA Journal, vol. 56, no. 8, pp. 3153-3166, 2018.

[21] T. W. Kim, S. J. Oh, and K. J. Yee, “The extension and validation of OpenFOAM algorithm for rotor inflow analysis using actuator disk model," Journal of the Korean Society for Aeronautical \& Space Sciences, vol. 39, no. 12, pp. 1087-1096, 2011.

[22] P. E. M. Rethore and N. N. Sørensen, "Actuator disc model using a modified Rhie-Chow/SIMPLE pressure correction algorithm. Comparison with analytical solutions," in 2008 European Wind Energy Conference and Exhibition, Brussels, Belgium, March-April 2008.

[23] http://www.openfoam.com.

[24] E. Robertson, V. Choudhury, S. Bhushan, and D. K. Walters, "Validation of OpenFOAM numerical methods and turbulence models for incompressible bluff body flows," Computers \& Fluids, vol. 123, pp. 122-145, 2015.

[25] R. I. Issa, "Solution of the implicitly discretised fluid flow equations by operator-splitting," Journal of Computational Physics, vol. 62, no. 1, pp. 40-65, 1986.

[26] S. V. Patankar and D. B. Spalding, "A calculation procedure for heat, mass and momentum transfer in three-dimensional parabolic flows," in Numerical prediction of flow, heat transfer, turbulence and combustion, pp. 54-73, Elsevier, 1983.

[27] A. T. Conlisk, "Modern helicopter aerodynamics," Annual Review of Fluid Mechanics, vol. 29, no. 1, pp. 515-567, 1997.

[28] G. J. Leishman, Principles of Helicopter Aerodynamics with CD Extra, Cambridge university press, 2006.

[29] F. Menter and T. Esch, Elements of Industrial Heat Transfer Predictions, 16th Brazilian Congress of Mechanical Engineering (COBEM), Brazil, 2001.

[30] D. B. Spalding, "A single formula for the "law of the wall"," Journal of Applied Mechanics, vol. 28, no. 3, pp. 455-458, 1961.

[31] Y. Han, Aerodynamic and Noise Analysis on Hovering Flight of High-Lift, Low-Noise Blade for Optimized Blade Design, Master thesis, Seoul National University, Republic of Korea, 2017.

[32] C. Woo, N. Park, S. Lee, and S. Lee, "Research on aerodynamic characteristics about fan-in-fin type tail rotor system using the CFD analysis," in Proceeding of The Korean Society for Aeronautical and Space Sciences Fall Conference, pp. 561-562, Republic of Korea, 2016.

[33] P. F. Yaggy and K. W. Mort, Wind-tunnel tests of two vtol propellers in descent, National Aeronautics and Space Administration, 1963.

[34] V. Srinivas, I. Chopra, D. Haas, and K. McCool, "Prediction of tail rotor thrust and yaw control effectiveness," Journal of the American Helicopter Society, vol. 40, no. 4, pp. 34-43, 1995.

[35] R. W. Prouty, Development of the Empennage Configuration of the YAH-64 Advanced Attack Helicopter, US Army Aviation Research and Development Command, 1983. 